\title{
Les sociétés au siècle de la santé
}

À la mémoire de Mirko Drager Grmek

Le droit à la santé apparaît, après le droit au bonheur au $X \mathrm{VIII}{ }^{\mathrm{e}}$ siècle et le droit à la liberté au XIXe, comme un nouveau droit de I'homme caractéristique du siècle qui vient de s'écouler. II est pourtant saisissable en filigrane, longtemps auparavant, à travers les revendications sociales de la Révolution de 1830, il a figuré dans la constitution de I'infortunée République de Weimar. C'est dans la fièvre de reconstruction de 1946 qu'il a été véritablement martelé par l'OMS. Celle-ci a défini la santé, dans une formule restée célèbre, comme un état de complet bienêtre physique, mental et social. Ainsi défini, le nouveau droit à la santé récapitule évidemment de multiples conditions qui se réfèrent à des revendications antérieures: logement, travail et liberté. Le slogan d'Alma Ata, "la santé pour tous", confirme I'universalité des nouvelles valeurs. La santé est devenue une référence incontournable permettant de comparer les sociétés et de porter un jugement sur elles.

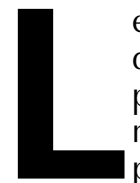

e droit à la santé, échappé des déclarations de principe pour venir s'inscrire concrètement dans les faits est saisi par des acteurs sociaux fort différents: à une extrême, l'état qui considère la sauvegarde de ce droit comme une de ses fonctions et tire parti de ce que Michel Foucault appelait la « gouvernementalité de la vie», à l'autre extrême, l'individu animé du souci de soi. Le corps médical a assuré historiquement la médiation entre I'un et l'autre. C'est lui qui a persuadé l'État de s'investir davantage dans la santé publique et qui a convaincu les patients de la pertinence du savoir scientifique pour soulager leurs maux. C'est lui qui à la fin du $X X^{\mathrm{e}}$ siècle a saisi entre ses mains un grand nombre de rouages de la vie publique et privée.

L'éducation du public, l'accès de tous aux sources d'information et d'imagerie ne vont-ils pas transformer la donne et permettre au citoyen d'affronter directement le pouvoir ? Ici I'anthropologie interroge I'histoire sur ce que certains n'hésitent plus à appeler l'avènement médical du sujet [1]. L'effacement de la médiation médicale serait conforme aux tendances individualisantes de l'ère post-moderne.

\section{La santé apparente de l'Occident}

Il y a quatre siècles, les Espagnols voyaient les Indiens comme des

\section{Anne-Marie Moulin}

A.M. Moulin: Cnrs, Département sociétéssanté, Institut de recherche pour le développement (IRD), 213, rue La Fayette, 75010 Paris, France.

sortes de bêtes fauves, animées d'une vie têtue et douées de caractéristiques différentes des autres hommes: leur crâne était plus dur, leur peau plus épaisse, les armes s'y émoussaient, les paroles s'y amortissaient. Aujourd'hui, c'est au tour de certains groupes indiens, durement éprouvés par les maladies, d'attribuer aux blancs une vitalité surnaturelle manifestée par leur état de santé florissant [2]. Elles ignorent l'impitoyable sélection naturelle qui s'exerça sur leurs envahisseurs dans le passé. Cette anecdote capture une vérité historique, I'atténuation contemporaine du sens de la maladie aiguë en Occident, la perte d'une expérience jouant un rôle considérable, la maladie comme apprentissage de la mort et de la résurrection.

L'expérience de la maladie est devenue marginale voire furtive. En Occident, il s'agissait autrefois d'un événement qui prenait importance et durée, mobilisait voisinage et famille, et s'associait fréquemment à un changement de mœurs, voire à une conversion, à tout le moins à des réflexions sur son "usage» qui pouvait être un «bon usage». Cette expérience se déroulait avec une grande solennité. Plus tard, des centenaires se rappelaient avec émotion avoir reçu plusieurs fois l'Extrême Onction.

Pensons au poète anglais John Donne à la Renaissance et à ses Devotions on Emergent Occasions, écrites pendant une épidémie de typhus: il y 
détaille par le menu les allées et venues de la fièvre, les variantes des bouillons et des clystères; I'arrivée du médecin est perçue comme celle d'un sauveur. Puis vient la convalescence, ces délicieuses retrouvailles avec les visages des proches, le retour à la vie, le réapprentissage de la marche, les premières sorties. Tout cela sur des semaines, au milieu de la stupeur d'une maisonnée qui vit au rythme du malade et se réveille comme la Belle au bois dormant.

Un siècle plus tard, si nous passons la Manche, et bien que le janséniste Pascal soit d'une autre obédience que le pasteur anglican, même tonalité avec la Prière pour le bon usage des maladies: la maladie est l'indispensable apprentissage de la mort dans "ce petit caveau (j'entends I'univers) ", où nous sommes logés.

$\mathrm{Au}$ XIX $\mathrm{X}^{\mathrm{e}}$ siècle, le voyage d'exploration est inextricablement lié à la maladie qui prend l'allure d'une initiation. En 1826, René Caillé arrive à Tombouctou pour s'effondrer aussitôt. Il passe plusieurs jours entre la vie et la mort, à la merci d'hôtes miséricordieux ou indifférents, percevant son entourage dans les brumes du délire. Pour beaucoup de voyageurs, les déplacements s'accomplissaient dans un état second: les alcools locaux, le haschich, la coca, I'opium, suivant le pays, fournissaient des appoints bienvenus pour affronter la fièvre, les blessures, la soif, la faim, mouches et moustiques. Les voyageurs, dilettantes ou aventuriers, gardent encore au début du $\mathrm{XX}^{\mathrm{e}}$ siècle la même logique (lire André Gide ou André Malraux [3, 4]).

"On ne sait plus être malade» [5]... L'expérience de la maladie aiguë est devenue plus rare, avec la disparition de la plupart des maladies de I'enfance: oreillons, rougeole, scarlatine, rubéole, qui jadis émaillaient inéluctablement la scolarité. Aujourd'hui, on n'attend guère pour réclamer un traitement qui abrège l'évolution et rende rapidement I'individu à son travail. La guérison est devenue "anachronique", comme dit joliment un philosophe américain [6].

La chirurgie a pris un caractère miraculeux et semble se substituer avantageusement au travail des forces naturelles. Toutefois, la brièveté de I'hospitalisation est trompeuse.
Depuis les mesures abrégeant la durée du séjour, les malades sont désorientés en constatant au retour chez eux que rien ne va plus comme par le passé. L'horloge du corps a pris I'habitude de retarder, il faut du temps pour retrouver le rythme antérieur. On parle beaucoup aujourd'hui, à propos du traitement du sida, de "reconstituer» le système immunitaire: on avait oublié le travail silencieux de remise en marche de l'organisme. Bref, ne faut-il pas réinventer la convalescence?

Une exception apparente à cette amnésie sociale de la maladie: la banalisation de I' «arrêt-maladie» et la revendication du droit à l'arrêt de courte durée. L'historien Marc Ferro suggère même que ce droit, aux yeux des travailleurs, passe désormais avant le droit de grève. II s'étonne que les ouvriers se soient démobilisés sur le chapitre des accidents du travail et maladies professionnelles, et ne poussent pas jusqu'au bout l'analyse de la responsabilité patronale (ou étatique) ([6], p. 95 et $s q q)$.

Pourtant dès le $x V I I I^{\mathrm{e}}$ siècle, à la suite de Bernardino Ramazzini, auteur du De artificum morbis [7], s'était allongée l'énumération des maladies liées aux activités artisanales ou préindustrielles. Au XIX ${ }^{\mathrm{e}}$ siècle, il n'est de profession qui n'ait son lot reconnu d'affections spécifiques, des polisseurs de mercure et d'argent aux dentelières et aux mineurs, et même aux écoliers, frappés de myopie, scoliose et constipation. Aujourd'hui, I'arrêtmaladie correspond souvent plus à un «ras le bol» qu'à une affection bien caractérisée. A l'analyse inquisitoriale des responsabilités, les syndicats préfèrent souvent une tolérance diffuse de la société à l'égard de l'absentéisme qui ne ressemble pas tout à fait à la maladie d'autrefois.

\section{Le retour du Dr Knock: tout le monde est malade}

Mais peut-être l'expérience de la maladie a-t-elle moins disparu qu'elle ne s'est diluée, et ses contours estompés. On n'attend plus l'apparition d'une symptomatologie bruyante pour prendre acte d'une pathologie, et l'idée prévaut d'intervenir en amont, suivant une démarche préventive, pour déceler le plus tôt possible la perturbation cachée d'un para- mètre biologique: la glycémie, les chiffres tensionnels. C'est, comme le titre un récent ouvrage sur le dépistage généralisé de I'hypertension [8], le retour du bon Dr Knock: au fond, tout le monde est malade, dans le règne du virtuel.

Du coup revient en force un problème jadis constant dans les préoccupations des pathologistes, celui de la réceptivité morbide, encore très présent dans la première génération des pastoriens. Aujourd'hui il refait surface, sous forme d'un faisceau de prédispositions ou $\mathrm{d}^{\prime}$ «immunosensibilités». Les compagnies d'assurances, orfèvres en matière de risque, essaient d'articuler leurs prévisions autour d'une carte génétique pour laquelle elles disposent d'un nombre croissant de paramètres.

A qui revient la gestion de ces malades virtuels ? N'est-ce pas à l'État, tirant parti de la gouvernabilité des corps? La tradition juridique hégélienne insistait sur la liberté absolue du sujet dans la disposition de son propre corps. Mais au $\mathrm{XIX}^{\mathrm{e}}$ siècle, la condamnation de l'esclavage amène l'État à limiter cette position libérale et à protéger I'individu contre lui-même en excluant le corps du patrimoine cessible par contrat. Parallèlement, et s'appuyant sur les nouveaux acquis scientifiques, I'État revendique l'exercice de la contrainte dans le domaine de la santé: le droit d'imposer les quarantaines, la vaccination, la déclaration de certaines maladies, etc.

Cette protection s'avère de nos jours insuffisante devant l'extension des pratiques de prélèvement des organes et cellules du corps, introduisant de nouveaux modes d'échange et de trafic. "L'homme aux cellules d'or» qui obtint compensation de la vente par un laboratoire américain d'une lignée de ses cellules, remettait en question le dogme de la non-patrimonialité du corps. Quand, dans I'homme, tout est bon ou presque, les os, la peau, la graisse, la cornée, les nerfs, où fixer les limites?

L'État a donc été amené à intervenir de plus en plus sur les corps humains [9], désormais intégrés dans un nouveau contrat social [10]. II interdit la vente des organes et des cellules, et la location d'utérus. II interdit aussi les modifications génétiques, si elles sont transmissibles à la descendance. 
Il vise ainsi à protéger non plus seulement les corps individuels, mais le corps en général saisi dans sa diversité et son potentiel de résistance et de survie de l'espèce, soulevant la question d'un nouveau sujet de droit, le « corps humain», peut-être impliqué dans une "nature» plus large, et qu'il s'agit alors de définir [11].

L'État n'a pas seulement assumé la mission de veiller sur la santé de ses citoyens, il a été amené à prendre en charge des maladies dans lesquelles il était directement impliqué.

Aux États-Unis, les combattants de la Guerre du Golfe se sont plaints d'un ensemble de symptômes pour lesquels ont tour à tour été invoqués le rôle des gaz de combat, celui d'une toxine bactérienne, I'uranium..., un syndrome mystérieux pour lequel l'état américain a ouvert une tumultueuse enquête et admis dans certains cas le droit à compensation. L'État français est aujourd'hui confronté aux mêmes questions.

En URSS, autour de Tchernobyl, toute une population a vécu un véritable traumatisme physique et psychique, le moindre n'étant pas la découverte des fraudes de l'État soviétique qui a exposé ses citoyens à un machinisme nucléaire vétuste et dangereux, et dissimulé consciemment des risques globalement connus. A sa création, le nouvel État ukrainien a bâti sa légitimité politique sur la reconnaissance officielle des dommages graves subis par la population. Les malades faisaient l'apprentissage d'une nouvelle citoyenneté à travers le montage difficile de leurs dossiers: plaintes somatiques et psychiques confrontées avec des critères de dosimétrie, des arguments génétiques et anatomopathologiques.

L'État ukrainien, de son côté, essayait de limiter le nombre des ayants droit, en jouant sur différents critères (dont la difficulté d'évaluer I'exposition réelle aux rayonnements). Il a favorisé l'individualisation, à côté du syndrome d'irradiation proprement dit, d'un syndrôme nouveau, la «dystonie végéto-vasculaire», conçu comme un leurre pour disperser les soupçons et diviser les esprits. Ce qui amène à envisager les nouveaux rapports de l'individu et de l'État et à souligner les ambiguités de la prise en charge opérée par ce dernier.

\section{Les miroirs du corps}

Mais quels sont les moyens pour le malade d'évaluer directement son état de santé ? Si la maladie ne joue plus que faiblement son rôle d'expérience du corps, la découverte scientifique de ce dernier est une aventure désormais largement partagée. De la radiographie au scanner, I'imagerie médicale a joué un grand rôle dans cette découverte.

La connaissance de l'anatomie était restée longtemps un secret maudit, en raison d'une longue histoire sulfureuse de dissections interdites ou réservées aux seuls initiés, de trafics de cadavres et de viols de cimetières (sur les avatars de la dissection et les trafics de cadavres jusqu'au XIX ${ }^{\mathrm{e}}$ siècle, voir entre autres [12, 13]). Elle commença à gagner le grand public au XIX ${ }^{\mathrm{e}}$ siècle, à la faveur d'ouvrages de vulgarisation qui lui consacrèrent de nombreuses planches en couleur, non sans un brin de voyeurisme.

C'est la radiographie, proche de la photographie, qui dédramatisa l'anatomie. Dans La Montagne magique de Thomas Mann [14], Clawdia Chauchat fait don de sa radio pulmonaire à I'amoureux Hans Castorp. II voit dans ce geste l'invite à partager la connaissance intime récemment acquise par la jeune femme de son propre corps.

Dans les années cinquante, au Yémen, pays jusqu'alors pratiquement interdit aux étrangers, le souverain, I'imam Yahia, fait venir auprès de lui un (une) radiologue. L'imam est fasciné par cet accès au mystère de son propre corps. Ignorant les dangers d'une exposition cumulative, il se satisferait volontiers d'une radio quotidienne qui le rassure sur sa santé.

Quarante ans plus tard, les anciens sujets de I'imam accèdent au privilège royal, à l'ère du scanner, et ressentent la brûlure de la modernité. Plusieurs scanners sont désormais opérationnels dans la capitale Sana'a, et les demandes affluent. Le prestige du scanner rappelle celui de la radiographie en Occident dans les années vingt ou dans le tiers monde dans les années cinquante, quand les patients réclamaient avec insistance le diagnostic par I’ «image »*. Si le Yémen a

\footnotetext{
* En arabe parlé, le même mot, "soura",
} désigne l'image et la radiographie. brûlé les étapes en introduisant en moins de vingt ans toute la gamme de l'imagerie, y compris les techniques les plus récentes, on peut suivre en Occident, à peine tempérée par le souci d'un risque, la même fascination de découvrir sa propre image, tout au long du $\mathrm{XX}^{\mathrm{e}}$ siècle.

Le scanner, lui, est à la fois rassurant et instructif. Comme le souverain yéménite, beaucoup de gens désireraient s'assurer tous les jours que leur scanner est sans faute. C'est un peu la reine dans Blanche Neige: $\mathrm{O}$ miroir, dis-moi que tout va bien ! L'échographie fotale a également déclenché un grand engouement, elle combine la découverte de soi et celle de l'Autre, et la fascination est double. Pour les grossesses non pathologiques, la Sécurité sociale a dû restreindre l'usage médical à trois séances, les autres sont pour l'album de famille.

Côté "savants", même fascination pour le miroir de soi, dans la recherche sur l'imagerie cérébrale [15]. Une sorte de jubilation envahit les neurophysiologistes, à I'habitude gens de laboratoire prudents et peu extravertis, à voir s'allumer, après injection $d$ 'un marqueur dans la circulation, telle région du cerveau en cas de stimulation. Si cet allumage est bien la preuve qu'il existe un lien indéniable entre la vascularisation du cerveau et l'activité mentale et psychologique, il est plus difficile d'y voir un déchiffrement de l'activité mentale et de son contenu. En d'autres termes, il est désormais possible de voir le penseur penser, sans pour autant en déduire ce qu'il pense ou même comment il pense. II n'est pas sûr que l'imagerie révèle de la pensée beaucoup plus que la célèbre statue de Rodin qui porte ce nom. La plaisanterie qui fait dire à la statue: "Mais où ai-je donc mis mon slip ? " pourrait s'appliquer à l'imagerie qui ne cède encore aucune information sur les pensers profonds.

Cela ne veut pas dire que le for intérieur soit définitivement opaque. II suffit de poser des garde-fous épistémologiques pour bénéficier de l'avancée incontestable qu'a représenté le fait d'intégrer dans un même champ le cognitif et le psychologique, I'anatomique et le mental, au sein d'une sphère analogique, ce qui veut dire qu'il y a un rapport suggestif entre l'un 
et I'autre, sans s'avancer au-delà de cette modeste appréciation. Reste à savoir si pour l'humanité ce serait une bonne chose de savoir non pas seulement ce que je pense, mais encore ce que I'autre pense. Ici s'opposent comme toujours les optimistes, ceux qui pensent que l'augmentation des savoirs suppose une vigilance et un code de bonnes pratiques mais demeure intrinsèquement un bien, et les esprits plus chagrins, marqués par le mythe du jardin de l'Éden, qui se préoccupent de poser fermement les bornes de la connaissance.

Quoi qu'il en soit, I'essor de l'imagerie sous toutes ses formes a eu pour conséquence de dévaloriser le savoir clinique et de contourner la médiation médicale assimilable à un simple geste technique. Le savoir sur le corps s'acquiert, si l'on peut dire, en tête à tête avec ce dernier

\section{Uniformisation et diversité des pratiques médicales}

S'appuyant sur la science médicale, et dépendant de ses experts, l'état contrôle désormais les procédures de la profession et tend à leur donner forme de règlements. En France et sur le modèle d'autres pays, il a récemment validé des procédures médicales de décision (les fameuses RMO ou références médicales opposables) sur le corps des citoyens, qui découlent de I'évaluation statistique des diagnostics et des thérapeutiques, et s'inspirent des conférences de consensus tenues à grande échelle, en un mouvement qui s'apparente à une "globalisation » du savoir. La médecine ne reposerait plus que sur l'evidence, terme anglais traduit malaisément par fait ou preuve. II ne s'agit pas de l'évidence cartésienne, où I'on ne doit selon l'auteur du Discours de la Méthode accepter que ce qui est clair et évident pour soi, mais du fait "hideux» ("the hideous fact» selon le mot d'Aldous Huxley), établi selon des règles rigoureuses par une armée d'observateurs. Le principe en fut proposé de manière systématique par un article du JAMA (Journal of the American Medical Association) en 1992 [16].

Cependant, une divergence se fait jour entre le projet d'une médecine evidence-based, corsetant le médecin dans des procédures qui ont force légale et lui sont opposables, et le désir de la société du maintien d'une certaine liberté de choix. Toutes les enquêtes des consommateurs soulignent la grande diversité des prescriptions et des itinéraires thérapeutiques [17]. La plupart des patients persistent à combiner des approches hétérogènes: kinésithérapie, chimiothérapie «naturelle», homéopathique ou exotique qui se reflètent dans la diversité des médecines proposées sur le marché. Cette diversité tend plutôt à s'étendre qu'à se restreindre en Occident, sur le modèle... du Tiersmonde, où elle est souvent officielle (Chine, Inde, Amérique latine), avec des universités qui enseignent côte à côte différents systèmes de théories médicales.

Cette diversité de prescription est-elle résiduelle ou futuriste ? Reflète-t-elle I'incapacité de la profession à se tenir au courant des dernières nouveautés, le désenchantement vis-à-vis du progrès, le désir de contenter un patient revendicateur, ou faut-il trouver une justification a priori de la pluralité des pratiques dans la diversité fondamentale des tempéraments et des constitutions? Les historiens de la médecine savante chinoise soulignent que pendant longtemps l'extrême hétérogénéité des pratiques n'a soulevé aucune difficulté, l'apprentissage de la médecine étant par définition lié aux relations personnelles de maître à élève, et la coutume étant d'adopter les techniques du maître élu en dernier ressort comme le plus savant. Or certaines techniques d'acupuncture, par exemple, sont actuellement intégrées à des essais standardisés par les chercheurs de I'université Johns Hopkins à Baltimore (Maryland, États-Unis), notamment pour les traitements de douleurs rebelles comme les migraines. Avec l'intégration de la Chine au monde occidental, se dessine le risque de décapiter une pluralité et une richesse essentielles, une réserve utile pour soulager la diversité des malades.

\section{L'autonomie médicale du sujet}

Le paradoxe de la modernité se présente alors en ces termes: alors que l'État de toute évidence étend son emprise sur le corps de ses sujets (lois de bioéthique entre autres), au point de les constituer en une "matière» gouvernable, il existe une tendance concomitante à restituer au sujet le droit de décider pour son corps. Au nom du nouveau savoir acquis par les individus qui les met plus à même de décider et leur ouvre la voie de l'indépendance, au nom comme toujours de bonnes et de mauvaises raisons, morales, politiques et... économiques. L'intervention des laïcs en médecine est en fait devenue plus importante qu'on ne croit couramment. Elle découle d'abord du fractionnement de la profession qui devient étrangère à elle-même. Les experts s'opposent entre eux. L'omnivalence du diplôme ne saurait cacher les conséquences sérieuses de l'hyperspécialisation qui sévit même au sein des spécialités. Tel chirurgien initié à la téléchirurgie ultra moderne par coelioscopie, qui s'introduit dans les viscères par une boutonnière élégante de quelques millimètres, s'avère, en cas d'hémorragie imprévue, mal à l'aise devant l'obligation de se replier vers des voies d'abord classiques, et passe la main à un chirurgien plus âgé qui opère "à I'ancienne». Devant cet émiettement des savoirs, le laïc tend à choisir luimême son praticien, comme la loi, au moins en France, l'y autorise, et par là même le type de thérapeutique, privilégiant l'esthétique ou la sécurité, la douleur ou le confort, la rééducation active ou les massages, la dialyse ou la transplantation. Il jouit, au moins dans les pays les plus riches, d'une réelle marge de manœuvre.

Cet avènement $d^{\prime}$ un nouveau «sujet » a été sans doute préparé par l'intervention croissante des associations de malades, qu'il s'agisse de maladies rares comme les myopathies ou d'épidémies inconnues comme le sida, frappant des groupes se sentant discriminés, en position défensive ou même offensive.

S'agissant de maladies rares, les malades ont eu le sentiment de posséder des connaissances non partagées sur les particularités de leur maladie, bref des savoirs cliniques inventifs. Leur expérience, parfois visualisée et décomposée par la caméra comme dans le cas des myopathies [18], leurs observations minutieuses au quotidien, collectées et mises au propre, ont fourni un corpus, suggéré des questions et des thèmes originaux de recherche. Disposant d'une réelle puissance finan- 
cière, les associations ont élaboré un discours autonome, entre science classique et vulgarisation, pour négocier les objets et les méthodes de la recherche privée et publique. Nous sommes très loin de la position d'otages mutuels dans laquelle se sont trouvés par exemple les hémophiles et leurs médecins [19]. Cette maturité amène à prendre des distances par rapport à la traditionnelle question de confiance: "dites-moi, docteur...", leitmotiv des ouvrages destinés au grand public, écrits par des médecins comme Les gènes de l'espoir de Daniel Cohen [20]. A ce titre, I'expérience étonnante de la grève entamée par des dialysés mécontents de leur centre (au péril de leur vie !), rapportée par la sociologue Renée Waismann [21], marque une étape symbolique dans une rupture comparable à celle de l'adolescence: quand le malade devient grand... Rupture avec la toute puissance et l'omniscience médicale.

La médicalisation croissante et la tentative de normalisation et de moralisation des comportements (sexualité, régime alimentaire, tabagisme...) ont provoqué un mouvement général de revendication face à la place indue prise par le jugement médical. La revendication d'autonomie a pris des formes multiples, du refus des soins à l'affirmation du droit à assumer un risque, ou à choisir un traitement alternatif ou non encore validé (sans parler des protocoles compassionnels). Pareil changement $d^{\prime}$ attitude enregistre $d^{\prime}$ ailleurs indirectement l'importance prise par la médecine dans le champ de la connaissance de soi. C'est parce que la médecine détaille toujours plus le faisceau complexe des déterminants hormonaux, physiologiques, nerveux, de la personne humaine, parce que le rapport du corps et de l'esprit s'approfondit, que le mouvement de revendication a pu prendre cette ampleur et ce caractère quasi-métaphysique.

Une revendication plus radicale encore s'est fait jour, impliquant le recours à la justice, avec le désir non seulement de voir compenser un dommage mais de pénaliser l'auteur de l'erreur, rebaptisée faute. Georges Canguilhem I'avait dit, I'homme de la Résistance. II avait évoqué la possibilité de convoquer le médecin non au tribunal de sa conscience mais au tri- bunal tout court, pour insister sur la perte d'immunité du praticien, la fin $d^{\prime}$ un privilège, après des siècles d'impunité. Le philosophe Alain Badiou a repris Canguilhem en termes plus brutaux: "et s'il (le médecin) refuse de comprendre, qu'on lui envoie la gendarmerie !» [22].

\section{Une compétence unique}

Mais le mouvement d'autonomisation du sujet a dépassé la simple contestation de la profession médicale, de son arrogance, de ses positions d'autorité excessive et de son monopole des ressources sanitaires dont dépend la concrétisation du «droit à la santé». Il a affirmé la compétence unique de l'individu à juger de ce qui était bon pour lui, le primat de son expérience subjective pour établir les normes de la vie.

Le sida est devenu aujourd'hui une maladie chronique qui jette une lumière nouvelle sur les problèmes liés à l'adoption de traitements de longue durée, complexes et parfois difficiles à gérer au quotidien. II a permis de mieux comprendre les phénomènes d'échappement à l'observance et la nécessité d'ajuster des programmes personnels tenant compte de la qualité de vie et des normes particulières à chacun, y compris en matière de risque consciemment affronté. La notion de qualité de vie a d'ailleurs fait l'objet d'une méthodologie nouvelle avec constitution d'une véritable spécialité, avec des études sur la qualité de vie des cancéreux, des dialysés, des handicapés de toutes sortes. II s'agit moins de définir le mode de vie de malades que les différentes modalités de la vie tout court [23].

En liaison avec ce qui a été dit plus haut sur la dissolution ou la dilution de la maladie dans la société occidentale du XXe siècle, la diversité des maladies est vue non plus seulement comme un phénomène à réduire et à combattre, mais comme une manière d'inventorier le corps et de réfléchir sur lui. C'est en ce sens que les sciences sociales sont appelées à intervenir pour une diversification et une meilleure adaptation des soins.

L'autonomie du sujet qui s'esquisse tend à dissoudre les limites de la maladie, les bornes entre le fou et le sage, ou le normal et le pathologique. Elle tend à disqualifier le discours médical, en oubliant parfois ce qu'elle lui doit: c'est parce que les hôpitaux proposent des techniques sophistiquées de survie ou de reconstruction de la "carcasse», qu'il est possible de contester couramment aujourd'hui les modalités de leur intervention. C'est en tenant compte du savoir véhiculé par la biologie, la radiologie, la psychiatrie ou la neurologie qu'on rediscute une définition de la santé, plus globale, qui prendrait en compte la singularité de l'individu. L'affimation par la biologie (immunologie, génétique...) de cette singularité, a certes été rendue acceptable par une longue tradition de philosophie du sujet passant par Descartes et tant d'autres, mais inversement est venue lester de savoir expérimental une intuition fondamentale [24].

Cette autonomie n'a pas été systématiquement combattue par l'État qui bien au contraire a favorisé dans certains cas la thèse de la responsabilité individuelle. Alors que les médecins se réjouissaient, depuis le XIX $X^{e}$ siècle, d'avoir arraché les malades aux griffes des Églises et libéré leurs consciences d'une faute imaginaire, voici qu'avec l'avènement potentiel du sujet en matière de décision médicale un nouveau type de culpabilité s'insinue: la responsabilité. L'État admet le besoin d'autonomie du sujet, mais l'instrumentalise à son tour en poursuivant ses objectifs propres: abaissement des coûts, culpabilisation dans l'engagement dans des conduites pathogènes: choix sexuels, prise de risque, non compliance, etc. Quelle est la faille individuelle qui a fait basculer un équilibre biologique instable? Quel gène délétère explique-t-il les résistances inégales à l'infection? Quelle immortalité sommes-nous en train de construire et à quel prix ?

La gestion partagée de la maladie peut aboutir à satisfaire les vœux des économistes et à déplacer le fardeau de la gestion des épaules des praticiens sur celles des "usagers de soins». Pareille ambivalence se retrouve dans l'attitude de l'État en matière de médecine prédictive. En matière de diagnostic prénatal, après consultation et information aussi complète que possible, l'individu se voit restituer sa capacité pleine et entière de décision. Pour éviter toute dérive totalitaire, l'État se désengage et pratique l'eugénisme à la petite semaine, en laissant à ses citoyens le 
choix de leur stratégie reproductive. La reconnaissance de l'autonomie du sujet en matière médicale ne va donc pas sans quelques effets pervers.

Où les différends entre l'individu, la société et l'État vont-ils désormais se régler? Tendent-ils à être plus graves et plus fréquents en raison de l'extension de la médicalisation, de l'intervention accrue de l'État dans le domaine de la santé publique, de la possibilité des employeurs, des compagnies d'assurance, d'imposer des normes, d'exiger des informations, de normaliser des comportements? Le conflit passe maintenant en partie devant les comités d'éthique dont la composition est plus ouverte que par le passé et intègre des individus appartenant à tout l'éventail des professions de santé, voire à des secteurs de la société civile éloignés du médical. Les comités ont défini des espaces de négociation où, au lieu de tenir pour acquise la moralité de la profession médicale, ils font entendre une parole, jugée jusqu'alors au chapitre de l'information du dossier et non au titre du jugement, celle du sujet malade.

\section{Conclusions}

L'autonomie médicale croissante du sujet, favorisée par l'évolution de la profession, renforcée par le progrès scientifique en immunologie, en endocrinologie, en génétique, renvoie au thème chatoyant $d$ 'une transparence du soi, faussée par toutes les informations apportées par la médecine. Elle est pour beaucoup synonyme de libération et aussi de vérité, signe dans la direction d'un accomplissement plénier du droit à la santé. Reste qu'un tel avènement peut correspondre aussi à une stratégie adoptée par la société à la recherche d'un nouvel équilibre économique et politique.

Mais c'est précisément sur les termes de vérité que se jouent les guerres du sens. La vérité médicale, dirait Louise Lambrichs [25], fort bien ! Mais de quelle vérité s'agit-il ? De la vérité objective, accumulation d'informations médicales énoncées en quel langage et pour qui? De la vérité subjective, du côté de l'intuition et de I'expérience singulière? Le jugement du sujet sur son corps propre, dégagé de la médiation des professionnels, permet-il d'aller très au-delà des infor- mations scientifiques? L'information actée et reçue ne le délivre pas nécessairement d'autres types d'obstacles, illustrés par exemple par l'inconscient freudien. La transparence du corps est un mythe, et l'imagerie ne triomphe pas de l'opacité du corps. La médecine n'échappe pas au sort des autres sciences, elle ouvre sans cesse de nouveaux labyrinthes et de nouveaux registres à l'imaginaire, d'où ce mélange de visions fulgurantes et de pulsions archaïques qui caractérise la modernité du corps. Le vrai n'est au fond qu'une des formes du possible. A l'aube du $x x^{\mathrm{e}}$ siècle, de nouveaux savoirs du corps sont en voie de construction, à la fois verticale et horizontale, personnelle et collective, relançant la dialectique entre savoir et pouvoir, liberté et solidarité

\section{Remerciements}

Je remercie Adriana Petryna, Volker Scheid, Marcela lacub, Patrick Triadou et Charles Moulin pour des informations stimulantes sur leurs recherches en cours.

\section{RÉFÉRENCES}

1. Gagnon E. L'avènement médical du sujet. Sci Soc Santé 1998; 16: 49-74.

2. Buchillet D. Perles de verre, parures de blancs et " pots de paludisme ». Journal de la Société des Américanistes 1995; 81 : 181 206.

3. Gide A. Voyage au Congo. Paris: Gallimard, 1925.

4. Malraux A. La voie royale. Paris: Grasset, 1930.

5. Beaune JC. Savoir être malade, maladie et images de la maladie: 1790-1990. Aubenas: Cahiers de la villa Gillet, Circé, 1995: 6.

6. Ferro M. Les sociétés malades du progrès. Paris: Plon, 1998.

7. Ramazzini B. De artificum morbis. Genève, 1770.

8. Corvol P, Postel-Vinay N. Le retour du Dr Knock. Paris : Odile Jacob, 1999.

9. Moulin AM. La crise éthique de la transplantation d'organes. A la recherche de la "compatibilité» culturelle. Diogène 1995; 172: 76-96.

10. Serres M. Le contrat naturel. Paris: Juillard, 1990.

11. Hermitte MA. Le concept de diversité biologique et la création d'un statut de la nature. In: Edelmann B, Hermitte MA, eds. L'homme, la nature et le droit. Paris: Ch. Bourgeois, 1988.

12. Sawday J. The body emblazoned. London: Routledge, 1995.
13. Richardson R. Death, dissection and the destitute. New York: Penguin Books, 1989.

14. Mann T (1922). La montagne magique. Paris: Fayard, 1961.

15. Le cerveau et les images. Annales d'Histoire et de Philosophie du vivant $2000 ; 3$.

16. Evidence-based Medicine Group. Evidence-based medicine: a new approach to teaching the practice of medicine. JAMA $1992 ; 268: 2420-5$.

17. Bouchayer F. Les usagers des médecines alternatives, Revue Française des Sciences Sociales 1986; numéro hors série: 104-16.

18. Rabeharisoa V, Callon M. L'implication des malades dans les activités de recherche soutenues par I'Association française contre les myopathies. Sci Soc Santé 1998; 16: 41-64.

19. Carricaburu D. L'hémophilie au risque de la médecine. Paris: Anthropos, 2000.

20. Cohen D. Les gènes de I'espoir. Paris: Robert Laffont, 1995.

21. Waisman R. Protest movement in a private clinic. An analysis of a patients' strike. Social Science and Medicine 1985; 20: 129-32.

22. Badiou A. L'éthique. Essais sur la conscience du mal. Paris: Hatier,1993.

23. La qualité de la vie. Prévenir 1997; 32.

24. Moulin AM. Le dernier langage de la médecine. Paris: PUF,1991.

23. Lambrichs LL. La vérité médicale. Paris: Robert Laffont, 1993.

\section{$\mathbf{m} / \mathbf{S} 2000$ \\ Summary}

Societies in the era of the right to health

The $X X$ th century has seen the development of a new human right, the right to health. The paper is a historical survey of the management of this new right by contemporary societies. The medical profession ensured the mediation between the state, increasingly involved in public health, and the individual in his quest for a better life. Following the transformation of medical practices, on the one hand, the popularization of body knowledge and the development of medical imagery, on the other, the individual emerges as the ultimate reference for a selfcentered definition of health and witnesses a relative decline in the power of the medical profession.

\section{TIRÉS À PART}

A.M. Moulin. 